\title{
Revisiting the formaldehyde masers
}

\author{
D. J. van der Walt $\odot$ and L. L. Mfulwane
}

Centre for Space Research, North-West University, Potchefstroom 2520, South Africa

e-mail: johan.vanderwalt@nwu.ac.za

Received 16 July 2021 / Accepted 30 November 2021

\begin{abstract}
Context. The $4.8 \mathrm{GHz}$ formaldehyde $\left(\mathrm{H}_{2} \mathrm{CO}\right)$ masers are one of a number of rare types of molecular masers in the Galaxy. There still is not agreement on the mechanism responsible for the inversion of the $1_{10}-1_{11}$ transition and the conditions under which an inversion can occur, and therefore how to interpret the masers.

Aims. The aim of the present calculations is to explore a larger region of parameter space to improve on our previous calculations, thereby to better understand the range of physical conditions under which an inversion of the $1_{10}-1_{11}$ transition occurs. We also aim to understand recently published results that $\mathrm{H}_{2} \mathrm{CO}$ masers are radiatively pumped.

Methods. We solve the rate equations of the first 40 rotational levels of o- $\mathrm{H}_{2} \mathrm{CO}$ using a fourth-order Runge-Kutta method. We consider gas kinetic temperatures between 10 and $300 \mathrm{~K}, \mathrm{H}_{2}$ densities between $10^{4}$ and $10^{6} \mathrm{~cm}^{-3}$, and a number of different dust temperatures and grey-body spectral energy density distributions.

Results. We show that when using a black body radiation field the inversion of any transition will disappear as the kinetic temperature approaches the black-body radiation temperature since the system, consisting of the gas and radiation field, approaches thermodynamic equilibrium. Using a grey-body dust radiation field appropriate for Arp 220 we find that none of $1_{10}-1_{11}, 2_{11}-2_{12}$, and $3_{12}-3_{13}$ transitions are inverted for kinetic temperatures less than $100 \mathrm{~K}$. Our calculations also show that in theory the $1_{10}-1_{11}$ transition can be inverted over a large region of explored parameter space in the presence of an external far-infrared radiation field. Limiting the abundance of $\mathrm{H}_{2} \mathrm{CO}$ to less than $10^{-5}$, however, reduces the region where an inversion occurs to $\mathrm{H}_{2}$ densities $\gtrsim 10^{5} \mathrm{~cm}^{-3}$ and kinetic temperatures $\gtrsim 100 \mathrm{~K}$. We propose a pumping scheme for the $\mathrm{H}_{2} \mathrm{CO}$ masers which can explain why collisions play a central role in inverting the $1_{10}-1_{11}$ transition, and therefore why an external radiation field alone does not lead to an inversion.

Conclusions. Collisions are an essential mechanism for the inversion of the $1_{10}-1_{11}$ transition. Our results suggest that $4.8 \mathrm{GHz} \mathrm{H}_{2} \mathrm{CO}$ megamasers are associated with hot and dense gas typical of high mass star forming regions rather than with cold material. Although limiting the $\mathrm{H}_{2} \mathrm{CO}$ abundance to less than $10^{-5}$ significantly reduces the region in parameter space where the $1_{10}-1_{11}$ is inverted, it still is not clear whether this is the only reason why these masers are so rare.
\end{abstract}

Key words. masers - stars: formation - ISM: molecules - radio lines: ISM

\section{Introduction}

The $4.8 \mathrm{GHz}$ ortho-formaldehyde (hereafter $\mathrm{H}_{2} \mathrm{CO}$ ) $1_{10}-1_{11}$ masers belong to a small group of rare astrophysical masers. At present only eight Galactic high mass star forming regions with

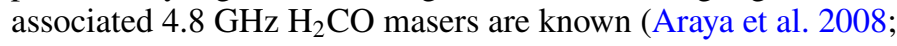
Ginsburg et al. 2015), compared with the more than $9006.7 \mathrm{GHz}$ methanol masers associated with high mass star forming regions detected in the methanol multibeam survey (Green et al. 2017). Since the $\mathrm{H}_{2} \mathrm{CO}$ masers seem to be exclusively associated with high mass star forming regions (Araya et al. 2015), the question arises of what is special about these star forming regions with associated $\mathrm{H}_{2} \mathrm{CO}$ masers compared to the majority of high mass star forming regions without associated $\mathrm{H}_{2} \mathrm{CO}$ masers.

High luminosity $4.8 \mathrm{GHz}$ megamaser emission has also been detected from the nuclear regions of three ultraluminous infrared (starburst) galaxies (Baan et al. 1986, 2017; Araya et al. 2004, 2008). Analogously to the question about the excitation of the $4.8 \mathrm{GHz}$ masers in a few Galactic high mass star forming regions, the same question arises for the extragalactic $\mathrm{H}_{2} \mathrm{CO}$ megamasers. All three known $\mathrm{H}_{2} \mathrm{CO}$ megamaser sources are also $\mathrm{OH}$ megamaser sources, which obviously raises the question of whether the two types of masers are excited in the same way.

Since the paper by Boland \& de Jong (1981), the question of the pumping of $\mathrm{H}_{2} \mathrm{CO}$ masers has regularly been raised over a period of about four decades. Recently, a number of authors (see e.g. Ginsburg et al. 2015; Lu et al. 2019) have remarked that the issue of the pumping of the $4.8 \mathrm{GHz} \mathrm{H}_{2} \mathrm{CO}$ masers is still unclear, which makes it difficult to interpret their observations. Further uncertainty also followed from the statement by Baan et al. (2017) that the masers are radiatively pumped, while van der Walt (2014) showed that an inversion of the $1_{10}-1_{11}$ transition can be achieved without the presence of an external far-infrared radiation field. van der Walt (2014) also found that no inversion could be found if collisions are switched off.

In view of the uncertainties in the pumping of the $\mathrm{H}_{2} \mathrm{CO}$ masers, we expanded the calculations of van der Walt (2014) to better cover the parameter space and thereby to obtain a more complete picture of the inversion of the $1_{10}-1_{11}$ transition. We also present the results of calculations aimed at understanding the results obtained with RADEX by Baan et al. (2017) and whether inversion of the $1_{10}-1_{11}$ transition can indeed be interpreted as being due to radiative pumping (by a black-body radiation field) as claimed by these authors. Following from the results, we propose a pumping scheme for the $\mathrm{H}_{2} \mathrm{CO}$ masers that explains why collisions play a central role in the inversion of the $1_{10}-1_{11}$ transition. For the present purposes we do not consider the effect of beaming, but focus only on the physical conditions under which an inversion can occur. 


\section{Molecular data}

Formaldehyde is a near prolate symmetric top molecule with the dipole moment along the $\mathrm{C}-\mathrm{O}$ axis (the $A$-axis). The energy levels are characterized by three quantum numbers, $J, K_{a}$, and $K_{c}$, with $J$ the total angular momentum, $K_{a}$ the projection of $J$ on the $A$-axis (the symmetry axis for a limiting prolate symmetric top), and $K_{c}$ the projection of $J$ on the $C$-axis (the symmetry axis for a limiting oblate symmetric top). In the case of $\mathrm{o}-\mathrm{H}_{2} \mathrm{CO}$, where the nuclear spins of the two hydrogen atoms are parallel, $K_{a}$ is odd. The slight asymmetry of the molecule causes each rotational level to be split into two energy levels (known as K-doublets) characterized by different values of $K_{c}$.

The energy level diagram for the first 40 levels of o- $\mathrm{H}_{2} \mathrm{CO}$ used in this work is shown in Fig. 1 for $K_{a}=1,3$. The energy levels and Einstein A coefficients for allowed radiative transitions were taken from the Leiden Atomic and Molecular Database (Schöier et al. 2005). The coefficients for collisions of o- $\mathrm{H}_{2} \mathrm{CO}$ with $\mathrm{o}-\mathrm{H}_{2}$ and $\mathrm{p}-\mathrm{H}_{2}$, as calculated by Wiesenfeld \& Faure (2013), were also obtained from the Leiden Atomic and Molecular Database. In all calculations the ratio of $\mathrm{o}-\mathrm{H}_{2}$ to $\mathrm{p}-\mathrm{H}_{2}$ was that for thermodynamic equilibrium at the specific kinetic temperature. For kinetic temperatures between 100 and $300 \mathrm{~K}$, a linear interpolation was used to produce collision rate coefficients for $10 \mathrm{~K}$ intervals instead of the $20 \mathrm{~K}$ intervals listed in the Leiden Atomic and Molecular Database.

Formaldehyde has six vibrational normal modes with the lowest at an energy of $1167 \mathrm{~cm}^{-1} \equiv 1679 \mathrm{~K}$ (Nikitin et al. 2021). This is significantly higher than the excitation energies associated with high mass star forming regions and it is therefore not necessary to consider excitation to the vibrational states.

\section{Numerical method}

The level populations were found by solving the well-known set of rate equations, which in the escape probability approach is given by

$$
\begin{aligned}
\frac{\mathrm{d} N_{i}}{\mathrm{~d} t}= & \sum_{j<i}\left[\left(-N_{i}+\left(\frac{g_{i}}{g_{j}} N_{j}-N_{i}\right) W \mathcal{N}_{i j}\right) \beta_{i j} A_{i j}\right. \\
& \left.+C_{i j}\left(N_{j} \frac{g_{i}}{g_{j}} e^{-E_{i j} / k T}-N_{i}\right)\right] \\
& +\sum_{j>i}\left[\left(N_{j}+\left(N_{j}-\frac{g_{j}}{g_{i}} N_{i}\right) W \mathcal{N}_{j i}\right) \beta_{j i} A_{j i}\right. \\
& \left.+C_{j i}\left(N_{j}-N_{i} \frac{g_{j}}{g_{i}} e^{-E_{j i} / k T}\right)\right] .
\end{aligned}
$$

Here $N_{i}$ is the number density in level $i, g_{i}$ the statistical weight of level $i, A_{i j}$ the Einstein A coefficient for spontaneous emission between levels $i$ and $j ; W$ is the geometric dilution factor for an external radiation field with a spectral energy distribution (SED) given by

$$
F_{v}\left(T_{\mathrm{d}}\right)=\left[1-e^{-\left(v / v_{0}\right)^{p}}\right] B_{v}\left(T_{\mathrm{d}}\right),
$$

and $\mathcal{N}_{i j}$ the photon occupation number for this field at frequency $v_{i j} ; C_{i j}=n_{\mathrm{H}_{2}} K_{i j}$ is the collision rate with $n_{\mathrm{H}_{2}}$ the $\mathrm{H}_{2}$ number density and $K_{i j}$ the collision rate coefficient. The fiducial frequency $v_{0}$ was taken as $3 \times 10^{12} \mathrm{~Hz} ; \beta_{i j}$ is the escape probability for which we used the expression for the large velocity gradient

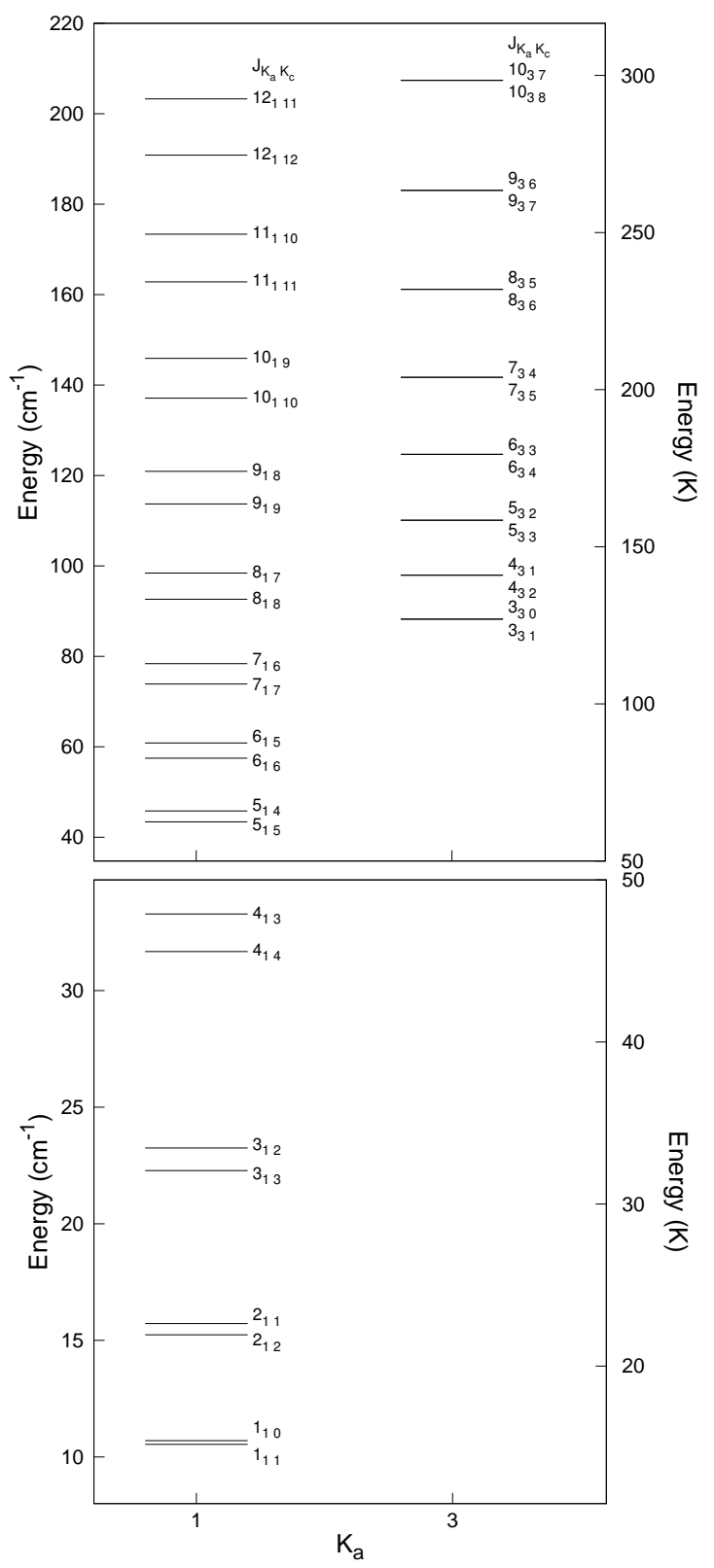

Fig. 1. Energy level diagram for the first 40 levels of orthoformaldehyde. Lower panel: first eight levels with $E<50 \mathrm{~K}$. Upper panel: levels with $E>50 \mathrm{~K}$. The energy scales are different on the $y$ axes for the lower and upper panels to better separate the doublet states at lower energies.

approximation

$\beta_{i j}=\frac{1-e^{-\tau_{i j}}}{\tau_{i j}}$,

with the optical depth given by

$\tau_{i j}=\frac{A_{i j}}{8 \pi}\left(\frac{c}{v}\right)^{3}\left(\frac{g_{i}}{g_{j}} x_{j}-x_{i}\right) \frac{N_{\mathrm{col}}}{\Delta v}$.

The quantities $x_{i}$ and $x_{j}$ are the fractional number densities of molecules in the upper level $i$ and lower level $j$, respectively; $N_{\text {col }}$ is the $\mathrm{H}_{2} \mathrm{CO}$ column density and $\Delta v$ the line width. The ratio $N_{\text {col }} / \Delta v$ is the specific column density. 
The rate equations were supplemented with the particle number conservation requirement

$N_{\mathrm{tot}}=X n_{\mathrm{H}_{2}}=\sum_{i} N_{i}$

with $X$ the abundance of $\mathrm{H}_{2} \mathrm{CO}$ relative to $\mathrm{H}_{2}$.

We solve the rate equations using a fourth-order Runge-Kutta method. The initial distribution of the level populations was a Boltzmann distribution with temperature equal to the average of the dust and kinetic temperatures. For a given $n_{\mathrm{H}_{2}}$ the calculation started with a small $\mathrm{H}_{2} \mathrm{CO}$ specific column density $\left(10^{5} \mathrm{~cm}^{-3} \mathrm{~s}\right)$ such that the system is completely optically thin in all transitions. An equilibrium solution for this initial specific column density was then found by letting the system evolve in time steps of $5 \mathrm{sec}-$ onds until the convergence condition $\left|N_{i}\left(t_{j+1}\right)-N_{i}\left(t_{j}\right)\right| / N_{i}\left(t_{j}\right)<$ $10^{-6}$ is reached for all levels. The specific column density is then increased by a small amount $(0.001 \mathrm{dex})$; the equilibrium solution for the previous value of the specific column density is used as the initial distribution. The process is repeated until a specific column density of $5 \times 10^{13} \mathrm{~cm}^{-3} \mathrm{~s}$ is reached or is stopped if $\tau_{4.8}>5$ before this specific column density is reached. If there is an inversion in the range of specific column densities, the maximum negative optical depth and the corresponding specific column density are recorded. If there is no inversion $\tau_{4.8}=0$.

\section{Results}

\subsection{The Baan et al. (2017) case}

We consider first Fig. 19 in Baan et al. (2017) which shows that inversion of the $1_{10}-1_{11}$ and $2_{11}-2_{12}$ transitions of $\mathrm{H}_{2} \mathrm{CO}$ can be achieved when locally the gas kinetic temperature is lower than the dust radiative temperature. Understanding the behaviour of the optical depth for different kinetic temperatures is essential before making any conclusions based on the shown behaviour.

Baan et al. (2017) obtained the results shown in their Fig. 19 using the online version of Radex (van der Tak et al. 2007). In particular, it should be noted that the online version of Radex uses an unshielded black-body radiation field (van Langevelde $\&$ van der Tak 2008) for the dust radiation. In Fig. 2 we show the dependence of the optical depth on the $\mathrm{H}_{2} \mathrm{CO}$ specific column density for the $1_{10}-1_{11}$ transition for $T_{\mathrm{k}}=10,20,30 \mathrm{~K} ; T_{\mathrm{d}}=$ $50 \mathrm{~K}$ (undiluted black body); and $n_{\mathrm{H}_{2}}=10^{4} \mathrm{~cm}^{-3}$ obtained with the above-described numerical method to solve the rate equations. Although there are quantitative differences in the optical depths when compared with Fig. 19 of Baan et al. (2017), the behaviour for the different values of the kinetic temperature is the same, which means that the inversion is largest for $T_{\mathrm{k}}=10 \mathrm{~K}$ and becomes smaller as the kinetic temperature increases to approach the dust temperature.

Figure 2 therefore confirms the result of Baan et al. (2017) that under the above-stated conditions the $1_{10}-1_{11}$ transition shows an inversion. We note that since the radiation field used is that of an undiluted black body, increasing the kinetic temperature to approach the radiation temperature brings the system closer to thermodynamic equilibrium. Thus, when $T_{\mathrm{k}}=T_{\mathrm{d}}$ the level populations follow a Boltzmann distribution, in which case a population inversion is not possible. We illustrate this further in Fig. 3 where we compare the level populations for the lower energy levels where inversions occur, when $T_{\mathrm{k}}=10,20,30,50 \mathrm{~K}$. The inversions for the different transitions are largest for $T_{\mathrm{k}}=$ $10 \mathrm{~K}$ and are seen to become smaller as $T_{\mathrm{k}}$ approaches $50 \mathrm{~K}$. For

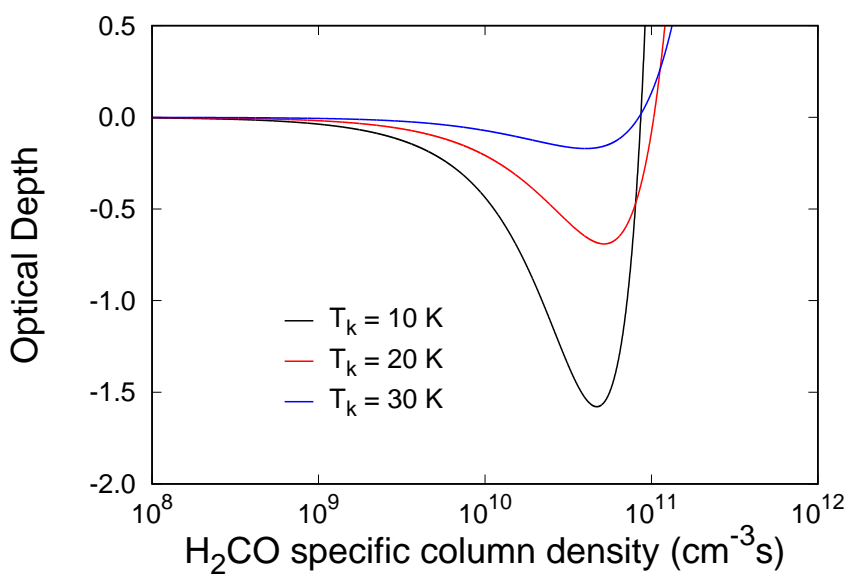

Fig. 2. 4.8 GHz optical depth as a function of the $\mathrm{H}_{2} \mathrm{CO}$ specific column density for $n_{\mathrm{H}_{2}}=10^{4} \mathrm{~cm}^{-3}$ and $T_{\mathrm{d}}=50 \mathrm{~K}$.

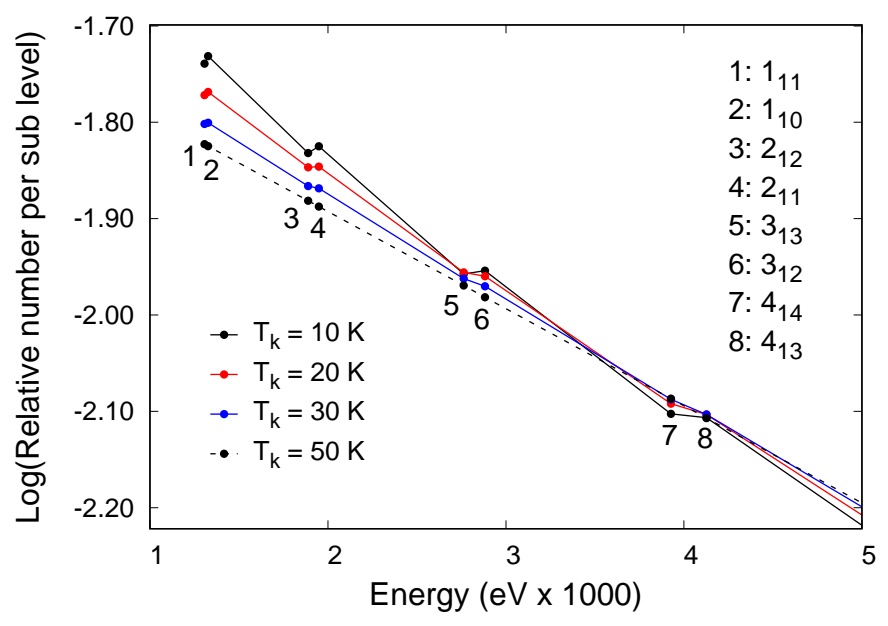

Fig. 3. Comparison of the level populations for levels $J_{K_{a}, K_{c}}$ from $1_{1,1}$ to $4_{1,3}$ for different kinetic temperatures when $n_{\mathrm{H}_{2}}=10^{4} \mathrm{~cm}^{-3}$ and the external radiation field is a black body with $T_{\mathrm{d}}=50 \mathrm{~K}$. The levels are numbered from 1 to 8 just below the case for $T_{\mathrm{d}}=50 \mathrm{~K}$.

$T_{\mathrm{k}}=T_{\mathrm{d}}=50 \mathrm{~K}$ there is no inversion and, consistent with thermodynamic equilibrium, the level populations follow a Boltzmann distribution with a temperature of $50 \mathrm{~K}$.

Still within the framework of the scenario proposed by Baan et al. (2017), we further examined the behaviour of $\tau_{4.8}$ in the $n_{\mathrm{H}_{2}}-T_{\mathrm{k}}$ plane for $10 \mathrm{~K} \leq T_{\mathrm{k}} \leq 300 \mathrm{~K}, 10^{4} \mathrm{~cm}^{-3} \leq n_{\mathrm{H}_{2}} \leq$ $10^{6} \mathrm{~cm}^{-3}$, and when dust emission is that of an undiluted black body at $50 \mathrm{~K}$. The result is shown in Fig. 4. It is seen that there are two distinct regions in the $n_{\mathrm{H}_{2}}-T_{\mathrm{k}}$ plane where the $1_{10}-1_{11}$ transition is inverted. The first is a small region with $T_{\mathrm{k}}<40 \mathrm{~K}$ and which corresponds with the results shown in Fig. 2. The second is a much larger region with $T_{\mathrm{k}} \gtrsim 130 \mathrm{~K}$ and $n_{\mathrm{H}_{2}} \gtrsim 10^{4.8} \mathrm{~cm}^{-3}$. The absence of an inversion for kinetic temperatures around $50 \mathrm{~K}$ is simply a manifestation of the system being close to thermodynamic equilibrium. As we show below, the second region corresponds to the region where inversion is due to collisions only (i.e. without the presence of an external dust infrared radiation field).

In the present case inversion of the $1_{10}-1_{11}, 2_{11}-2_{12}$, and $3_{12}-3_{13}$ transitions has been achieved using a black-body radiation field for the dust emission. In reality the dust radiation 


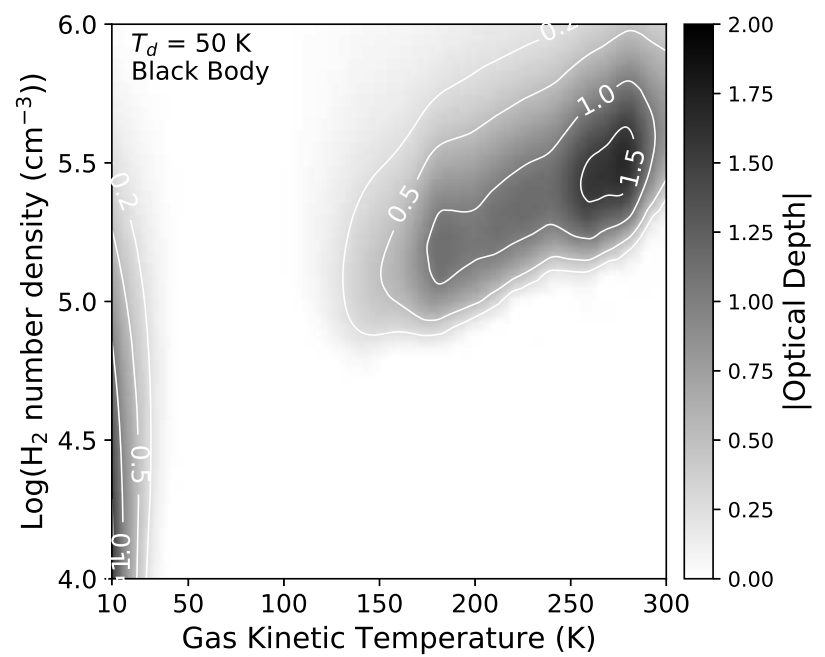

Fig. 4. Variation in the $4.8 \mathrm{GHz}$ optical depth in the $n_{\mathrm{H}_{2}}-T_{\mathrm{k}}$ plane when the spectral energy distribution (SED) of the external emission is given by an undiluted black body at $50 \mathrm{~K} . \tau_{4.8}=0$ means that there is no inversion.

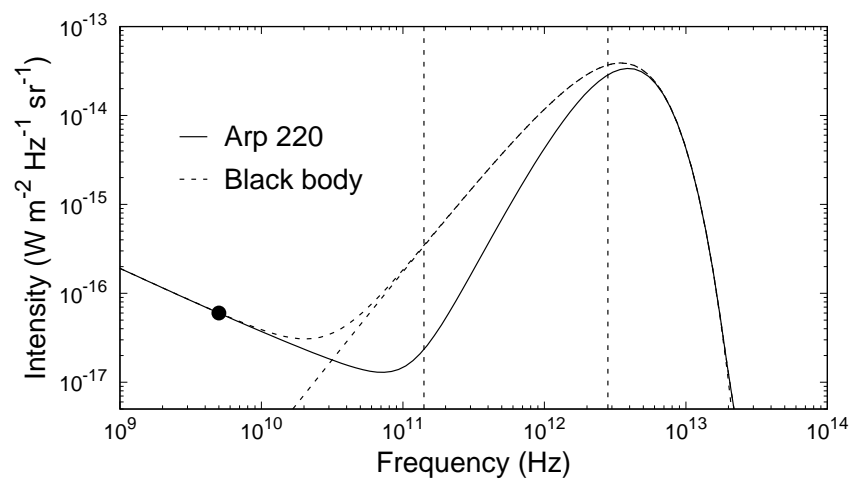

Fig. 5. Comparison of a black-body SED with the radio-to-far-infrared SED for Arp 220 (Yun \& Carilli 2002). The left vertical dashed line is at $140.8 \mathrm{GHz}$, which is the frequency for the $3_{13}-1_{11}$ transition. The right vertical dashed line is at $2808 \mathrm{GHz}$, which is the highest transition frequency for the 40 levels.

field is not that of a black body. Staying within the scenario presented by Baan et al. (2017) that the dust temperature is $\sim 50 \mathrm{~K}$, we used the radio-to-far-infrared SED of Arp 220 derived by Yun \& Carilli (2002). The SED of the dust emission is given by Eq. (2) with $T_{\mathrm{d}}=59 \mathrm{~K}$ and $p=1.15$ (Yun \& Carilli 2002) and is compared in Fig. 5 with that of a black body at the same temperature. In Fig. 6 we show the corresponding variation of $\left|\tau_{4.8}\right|$ in the $n_{\mathrm{H}_{2}}-T_{\mathrm{k}}$ plane. Comparison with Fig. 4 shows that while at higher densities and kinetic temperatures the shape of the region where there is an inversion of the $1_{10}-1_{11}$ transition is more or less the same, there is no inversion of the $1_{10}-1_{11}$ transition for $T_{\mathrm{k}} \lesssim 100 \mathrm{~K}$ in this case.

\subsection{Expanding the calculations of van der Walt (2014)}

van der Walt (2014) presented results of the inversion of the $1_{10}-1_{11}$ transition for a limited number of selected combinations of $T_{\mathrm{k}}, n_{\mathrm{H}_{2}}$, and $T_{\mathrm{d}}$ which represented only small parts of the parameter space. We now turn to presenting somewhat more general results covering $10 \mathrm{~K} \leq T_{\mathrm{k}} \leq 300 \mathrm{~K}$ and $10^{4} \mathrm{~cm}^{-3} \leq$ $n_{\mathrm{H}_{2}} \leq 10^{6} \mathrm{~cm}^{-3}$, where we consider the effect of having no external radiation field as a source of excitation and we investigate

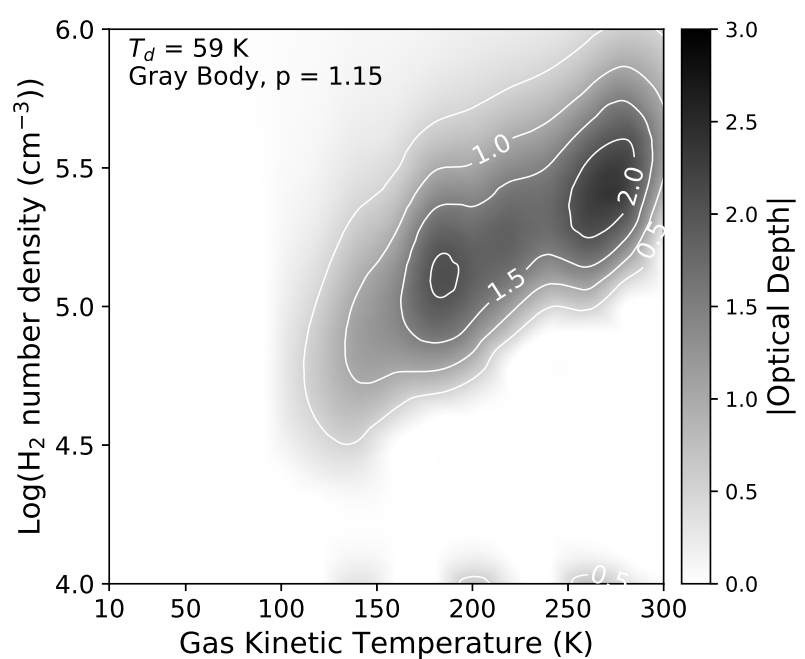

Fig. 6. Variation of $\left|\tau_{4.8}\right|$ in the $n_{\mathrm{H}_{2}}-T_{\mathrm{k}}$ plane when the SED of the dust emission is given by $F_{v}\left(T_{\mathrm{d}}\right)=\left[1-e^{-\left(v / v_{0}\right)^{p}}\right] B_{v}\left(T_{\mathrm{d}}\right)$ with $p=1.15$ and $T_{\mathrm{d}}=59 \mathrm{~K}$ as for Arp 220 (Yun \& Carilli 2002). The synchrotron and free-free components shown in Fig. 5 have also been included.

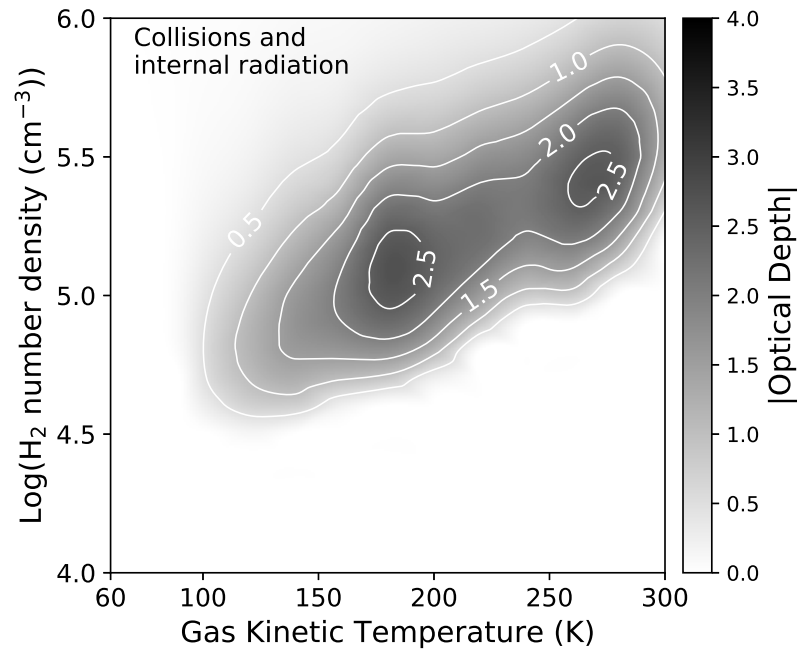

Fig. 7. Variation of $\left|\tau_{4.8}\right|$ in the $n_{\mathrm{H}_{2}}-T_{\mathrm{k}}$ plane when there is no external dust radiation field. Excitation is only through collisions and the internal radiation field.

the effect of an external radiation field on the inversion of the $1_{10}-1_{11}$ transition.

In Fig. 7 we show the behaviour of $\left|\tau_{4.8}\right|$ in the $n_{\mathrm{H}_{2}}-T_{k}$ plane when the external radiation field is switched off (i.e. excitation is due only to the internal radiation field and collisions). Inversion of the $1_{10}-1_{11}$ transition occurs for $T_{\mathrm{k}} \gtrsim 100 \mathrm{~K}$ and $n_{\mathrm{H}_{2}} \gtrsim 10^{4.5} \mathrm{~cm}^{-3}$. This result supports the results of van der Walt (2014) that the $1_{10}-1_{11}$ transition can be inverted without the presence of an external far-infrared radiation field. It does not, however, explain what role collisions play in the pumping of the masers.

With regard to the choice of dust and gas kinetic temperatures, it is noted that Araya et al. (2015) concluded that Galactic $\mathrm{H}_{2} \mathrm{CO}$ masers are exclusive tracers of high mass star forming regions. Various studies of Galactic high mass star forming regions have found dust temperatures to be greater than 50-60 K (see e.g. Kraemer et al. 2001; Barbosa et al. 2016; Lim \& De Buizer 2019). This also applies to higher resolution 

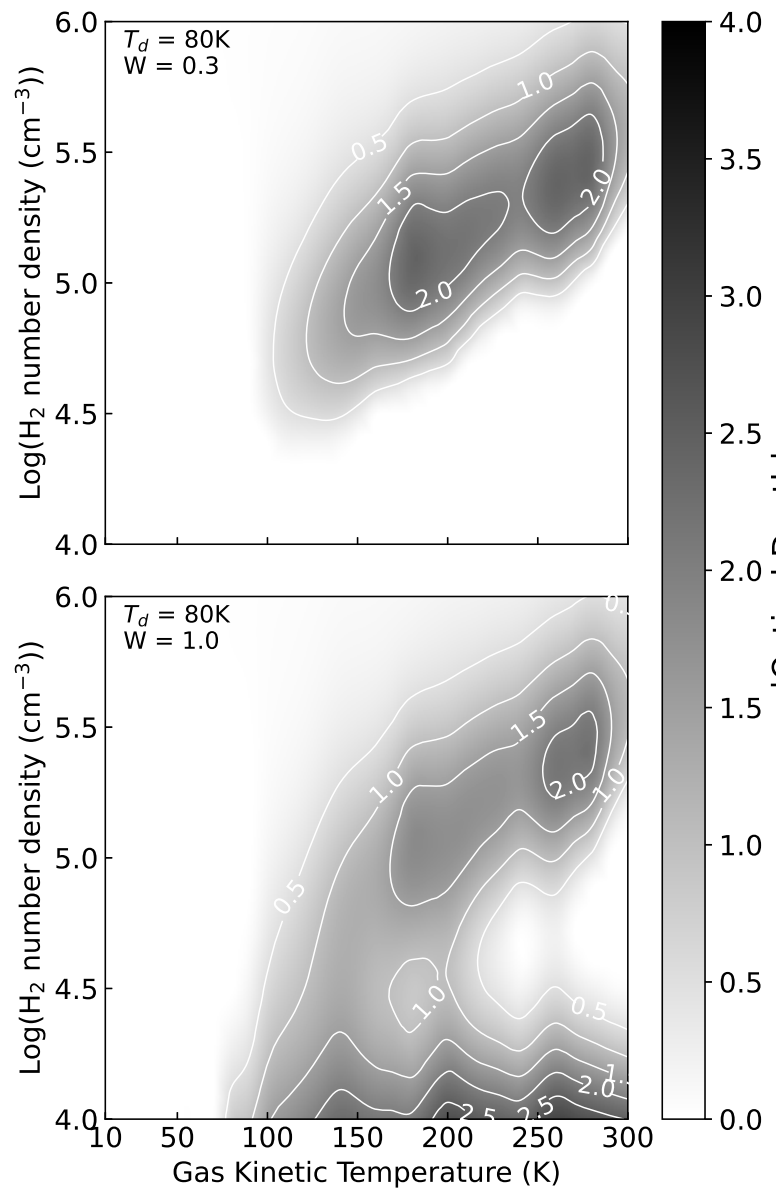

Fig. 8. Variation of $\left|\tau_{4.8}\right|$ in the $n_{\mathrm{H}_{2}}-T_{\mathrm{k}}$ plane when the SED of the dust emission is given by Eq. (2) with $p=1.15$ and $T_{\mathrm{d}}=80 \mathrm{~K}$. The synchrotron and free-free components shown in Fig. 5 have also been included. The upper panel is for $W=0.3$ and the lower panel for $W=1$.

observations of Arp 220 by Wilson et al. (2014), among others, who derived average dust temperatures of $80 \mathrm{~K}$ and $197 \mathrm{~K}$ for the eastern and western nuclei, respectively. Downes \& Eckart (2007) limits the intrinsic dust temperature in the western nucleus of Arp 220 to between 90 and $180 \mathrm{~K}$ based on the size and luminosity of the region. Scoville et al. $(2015,2017)$ derived a dust temperature $>100 \mathrm{~K}$ for the western nucleus of Arp 220 . We therefore considered three more cases with $T_{\mathrm{d}}=80,100$, and $150 \mathrm{~K}$ to illustrate the effects of higher dust temperatures and of geometric dilution. While presenting these results we acknowledge that the combinations of $T_{\mathrm{d}}, T_{\mathrm{k}}$, and $n_{\mathrm{H}_{2}}$ presented do not necessarily take the thermal balance between dust and gas into account.

In Figs. 8-10 we therefore show the behaviour of $\left|\tau_{4.8}\right|$ in the $n_{\mathrm{H}_{2}}-T_{\mathrm{k}}$ plane for $T_{\mathrm{d}}=80,100$, and $150 \mathrm{~K}$, respectively. The lower panels in these figures are for the case of an undiluted radiation field ( $W=1$ in Eq. (2)). The upper panels in Figs. 8 and 9 are for geometric dilution factors $W=0.3$, while the upper panel in Fig. 10 is for $W=0.1$. The effect of higher dust temperatures is interesting in that the region in the $n_{\mathrm{H}_{2}}-T_{\mathrm{k}}$ plane over which the $1_{10}-1_{11}$ transition is inverted changes significantly when $W=1$. In Figs. 6 and 7 inversion occurs basically for $T_{\mathrm{k}} \gtrsim 100 \mathrm{~K}$ and $n_{\mathrm{H}_{2}} \gtrsim 10^{4.5} \mathrm{~cm}^{-3}$, whereas for warmer dust the inversion occurs at lower $\mathrm{H}_{2}$ densities and also somewhat lower kinetic temperatures. An undiluted warmer dust radiation field also results in larger values of $\left|\tau_{4.8}\right|$ when compared to the case shown in Fig. 7 .

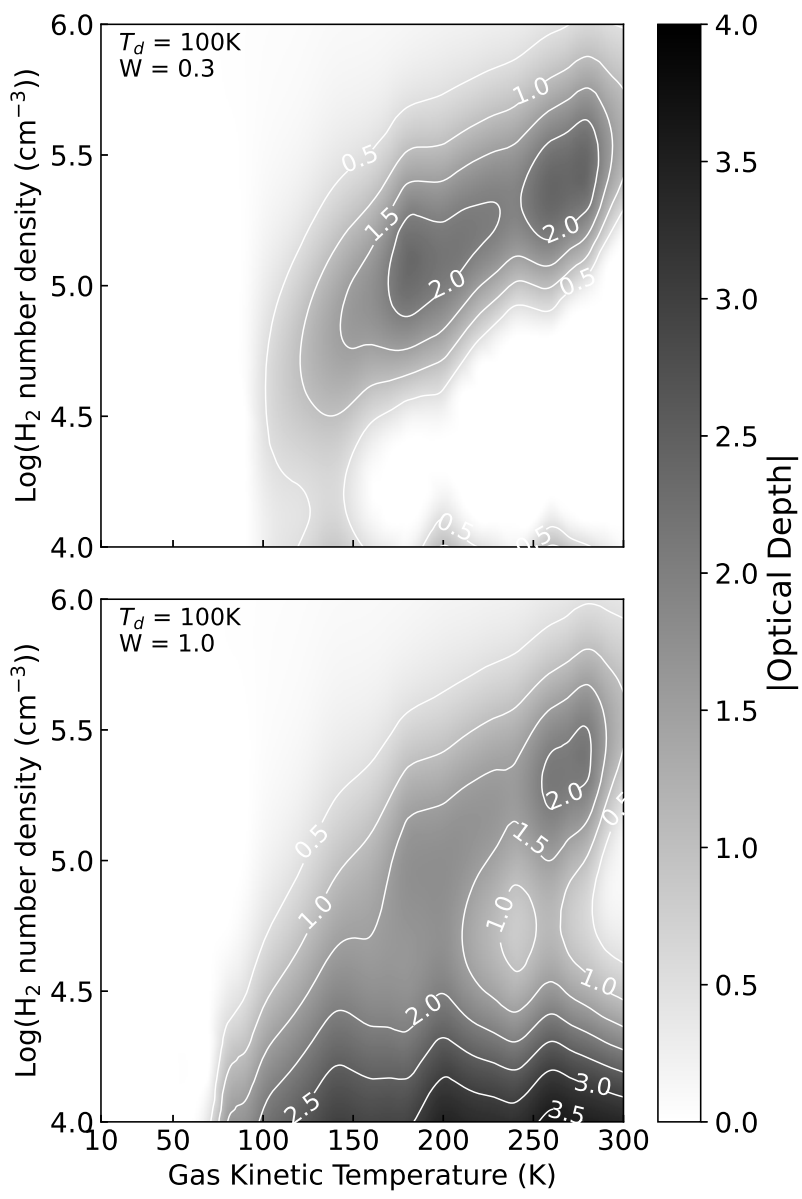

Fig. 9. Variation of $\left|\tau_{4.8}\right|$ in the $n_{\mathrm{H}_{2}}-T_{\mathrm{k}}$ plane when the SED of the dust emission is given by Eq. (2) with $p=1.15$ and $T_{\mathrm{d}}=100 \mathrm{~K}$. The synchrotron and free-free components shown in Fig. 5 have also been included. The upper panel is for $W=0.3$ and the lower panel for $W=1$.

On the other hand, significant dilution $(0.1-0.3)$ of the external radiation field in all three cases leads to a result very similar to that when collisions and the internal radiation field are the only mechanisms for excitation.

Closer inspection of Figs. 8 and 9 shows the presence of faint regularly spaced vertical regions of weak inversion of the $1_{10}-1_{11}$ transition. For example, the top panel of Fig. 9 shows that for $W=0.3$ the larger region of inversion connects to lower densities at $T_{\mathrm{k}} \sim 140 \mathrm{~K}$ and that regions of weak inversions also start to appear at $T_{\mathrm{k}} \sim 200 \mathrm{~K}$ and $260 \mathrm{~K}$. This results in the wavy nature of the contours for $n_{\mathrm{H}_{2}} \lesssim 10^{4.5} \mathrm{~cm}^{-3}$ seen in both Figs. 8 and 9. Test runs with smaller time steps, smaller steps in specific column density, and different convergence criteria were performed to check if this behaviour is due to the numerical method used. In all these cases the wavy behaviour of the optical depth was present. The exact reason for this behaviour of the optical depth is not clear and has not been investigated further.

In addition to the dilution factor, we finally consider the effect of the shape of the SED of the dust emission. In Fig. 11 we show in the upper panel the variation of $\left|\tau_{4.8}\right|$ for $T_{\mathrm{d}}=100 \mathrm{~K}$ when $p=0.5$ in Eq. (2) and in the lower panel when $p=2.0$. These two cases should be compared with the lower panel of Fig. 9 for which $p=1.15$. It can be seen that although the region over which the $1_{10}-1_{11}$ transition is inverted is more or less the same for the three values of $p,\left|\tau_{4.8}\right|$ is smaller over the whole region for $p=0.5$ and larger when $p=2.0$. In the Rayleigh-Jeans limit and 

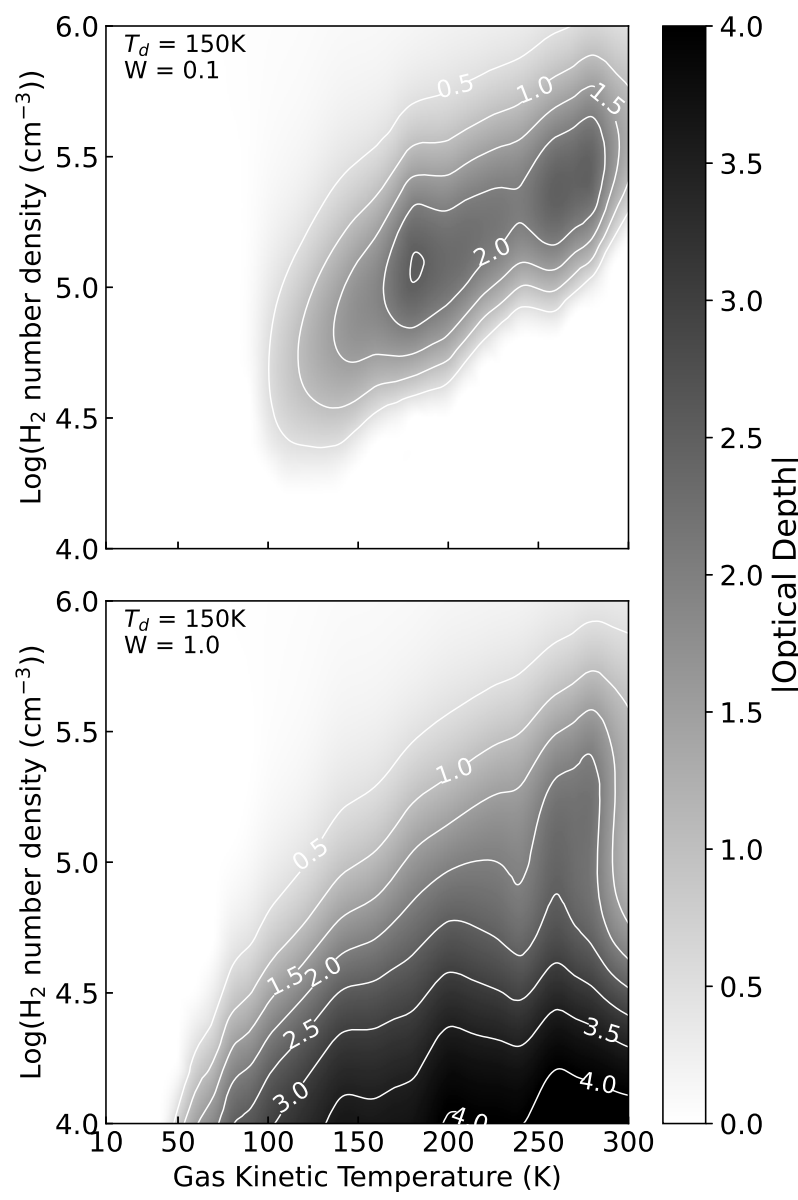

Fig. 10. Variation of $\left|\tau_{4.8}\right|$ in the $n_{\mathrm{H}_{2}}-T_{\mathrm{k}}$ plane when the SED of the dust emission is given by Eq. (2) with $p=1.15$ and $\mathrm{T}_{\mathrm{d}}=150 \mathrm{~K}$. The synchrotron and free-free components shown in Fig. 5 have also been included. The upper panel is for $W=0.1$ and the lower panel for $W=1$.

for $\left(v / v_{0}\right)^{p} \ll 1$ in Eq. (2), $F_{v}\left(T_{\mathrm{d}}\right) \propto v^{2+p}$. When $p=0, F_{v}\left(T_{\mathrm{d}}\right)=$ $0.63 B_{v}\left(T_{\mathrm{d}}\right) \propto v^{2}$, which is the flattest possible SED in the Rayleigh-Jeans regime. Except for the factor of 0.63 , the result presented in Fig. 4 corresponds to the case when $p=0$. Therefore, considering Figs. 4, 9, and 11, there is a clear dependence of $\left|\tau_{4.8}\right|$ on the behaviour of the SED at frequencies lower than the turnover frequency: flatter power laws (smaller $p$ ) result in smaller values of $\left|\tau_{4.8}\right|$ compared to steeper power laws (larger $p$ ).

As a last result we note that by following exactly the same calculational procedure as when collisions are included, no evidence could be found for the $1_{10}-1_{11}$ transition being inverted by the external dust radiation field (i.e. when collisions are switched off).

\section{Discussion}

Inspection of Figs. 7-11 shows some interesting behaviour of the $1_{10}-1_{11}$ maser which had not been seen or anticipated in the results of van der Walt (2014). Although the basic conclusions of van der Walt (2014) are, in a limited sense, still valid, this study shows that the presence of a far-infrared dust radiation field significantly affects the region in the $n_{\mathrm{H}_{2}}-T_{\mathrm{k}}$ plane where an inversion can occur. This obviously calls into question the con-

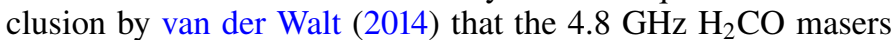
are strictly collisionally pumped. If a far-infrared radiation field has such a significant effect on the $4.8 \mathrm{GHz}$ masers, then what is the role of collisions, especially if no inversion seems to occur

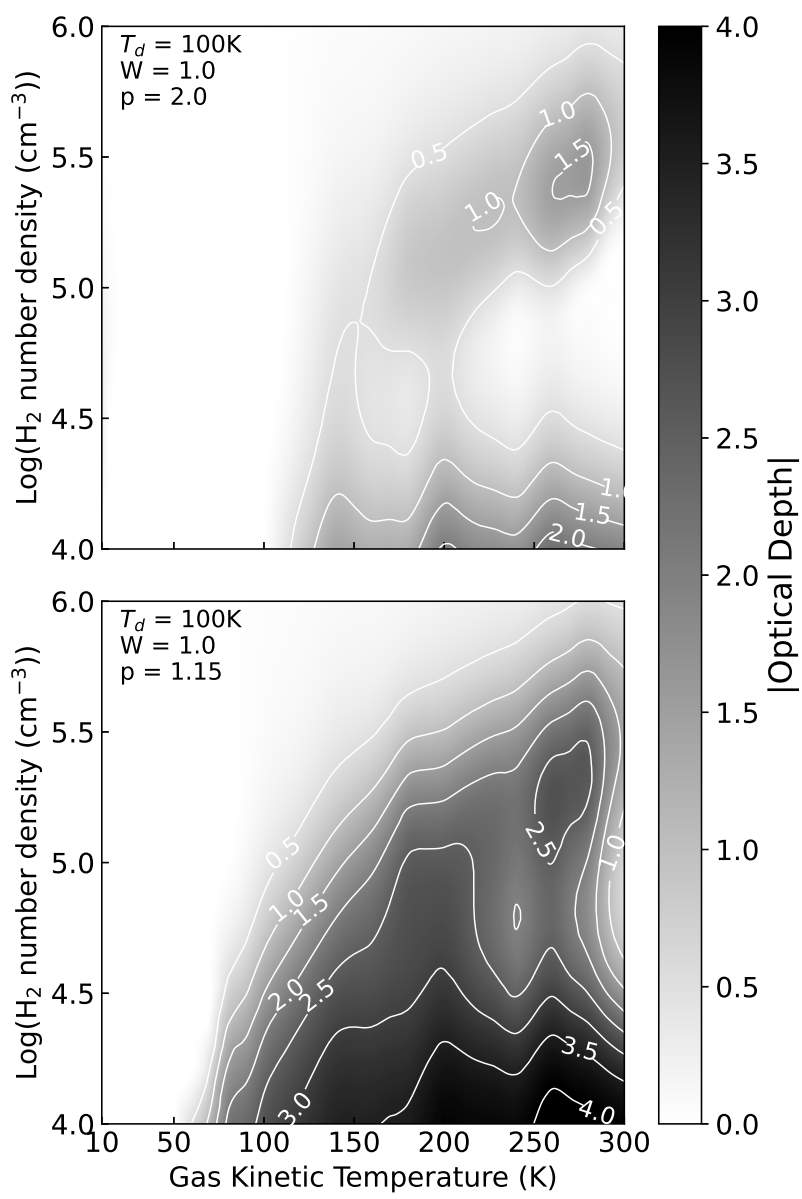

Fig. 11. Comparison of the variation of $\left|\tau_{4.8}\right|$ in the $n_{\mathrm{H}_{2}}-T_{\mathrm{k}}$ plane when the SED of the dust emission is given by $F_{v}\left(T_{\mathrm{d}}\right)=\left[1-e^{-\left(v / v_{0}\right)^{p}}\right] B_{v}\left(T_{\mathrm{d}}\right)$ with $p=0.5$ in the upper panel, $p=2.0$ in the lower panel, and $T_{\mathrm{d}}=$ $100 \mathrm{~K}$ in both cases.

when collisions are switched off? There are two theoretical limiting results which indicate that collisions play a central role in the inversion of the $1_{10}-1_{11}$ maser. The first is that an inversion can be obtained without the presence of an external far-infrared radiation field, and the second that no inversion is found if collisions are switched off even if an external far-infrared radiation field is present. These two limiting cases, as well as the other results, must be the consequence of a specific pumping scheme for the formaldehyde masers. We propose a possible pumping scheme and attempt to explain the results within this framework.

\subsection{Proposed pumping scheme}

To create a population inversion for the $1_{10}-1_{11}$ transition, it is necessary that the excitation rate out of the $1_{11}$ state be faster than the rate at which the $1_{10}$ state is populated through downward transitions from higher energy states. Inspection of the Einstein A coefficients shows that the fastest radiative downward route to $1_{10}$ is through the upper levels of the doublet states of $K_{a}=1$ (e.g. $7_{16} \rightarrow 6_{15} \rightarrow 5_{14} \rightarrow 4_{13} \rightarrow 3_{12} \rightarrow 2_{11} \rightarrow 1_{10}$ ). It therefore seems that the pumping scheme must be such that there is a route and mechanism whereby molecules can be excited to the upper levels of the doublet states. Direct upward radiative excitation out of $1_{11}$ to the upper levels of the doublet states is limited to $\Delta J=0,1, \Delta K_{c}= \pm 1$ and must therefore start with excitation first to $1_{10}$. However, this transition is very slow. For example, for 
an undiluted dust radiation field as in Eq. (2) with $T_{\mathrm{d}}=100 \mathrm{~K}$ and $p=1.15$ (the case for Arp 220; the exact value is not important for this example), the radiative transition rate for $1_{11} \rightarrow 1_{10}$ is only $7.4 \times 10^{-10} \mathrm{~s}^{-1}$. On the other hand, the corresponding rate for $1_{11} \rightarrow 2_{12}$ is $2.9 \times 10^{-5} \mathrm{~s}^{-1}$. Upward radiative excitation out of $1_{11}$ via $2_{12}$ to the lower levels of higher $J$ doublet states (e.g. $\left.3_{13}, 4_{14}, 5_{15}\right)$ is therefore much faster than via $1_{10}$ to the upper levels of higher $J$ doublet states (e.g. $2_{11}, 3_{12}, 4_{13}, 5_{14}$ ).

To populate the $1_{10}$ state, and since radiative excitation out of $1_{11}$ via $1_{10}$ to the upper levels of the doublet states is very slow, it is necessary to transfer molecules from the lower levels of the doublet states to the upper levels of the doublet states, which then provides a fast radiative decay route to $1_{10}$. However, radiative transitions from the lower levels of the doublet states to the associated upper levels are very slow compared to radiative transitions between the lower levels of the doublet states. For example, the radiative transition rate for $5_{15} \rightarrow 5_{14}$ is $6.2 \times 10^{-8} \mathrm{~s}^{-1}$ for $T_{\mathrm{d}}=100 \mathrm{~K}$ and $p=1.15$. On the other hand, the radiative transition rate for $5_{15} \rightarrow 4_{14}$ is $1.2 \times 10^{-3} \mathrm{~s}^{-1}$ and $4.2 \times 10^{-4} \mathrm{~s}^{-1}$ for $5_{15} \rightarrow 6_{16}$. The implication is that if a molecule is excited from $1_{11}$ to $2_{12}$ (the lower doublet state for $J=2$ ), further upward and downward radiative excitations will only be within the ladder of lower level doublet states.

We illustrate the above in Fig. 12, where radiative and collisional rates for different transitions are compared for the case when $n_{\mathrm{H}_{2}}=10^{4.25} \mathrm{~cm}^{-3}, T_{\mathrm{k}}=180 \mathrm{~K}, T_{\mathrm{d}}=100 \mathrm{~K}$, and $p=1.15$. In the upper panel we show the radiative rates for upward transitions $\left(\Delta J=+1, \Delta K_{c}=+1\right.$, black squares) between the lower levels of the doublet states, the corresponding downward rates $\left(\Delta J=-1, \Delta K_{c}=-1\right.$, filled red dots $)$, and the upward radiative rates from the lower levels of the doublet states to the corresponding upper levels $\left(\Delta J=0, \Delta K_{c}=-1\right.$, e.g. $5_{15} \rightarrow 5_{14}$, open circles) for $K_{a}=1$. Radiative rates between the $K_{a}=1$ and $K_{a}=3$ ladders are so low that radiative transitions between the ladders can be ignored. Also shown are the total collision rates out of the lower level of the doublet states to any other level (blue triangles), and more specifically the collision rates from the lower levels of the doublets to any of the upper level doublet states (red diamonds). As can be seen, the upward radiative rates between the lower levels of the doublet states (black squares) are orders of magnitude higher than the radiative rates from the lower levels of the doublet states to their associated upper levels of the doublet states (open circles). This illustrates the point made above that if a molecule is excited (radiative or collisionally) from $1_{11}$ to the $2_{12}$ state, further radiative transitions will only be within the ladder of the lower levels of the doublet states and that radiative transfer of molecules from the lower levels of the doublet states to their associated upper levels is not effective. On the other hand, under the given physical conditions, collision rates from the lower levels of the doublet states to any of the upper levels of doublet states (red diamonds) are significantly higher than the corresponding radiative rates. This is also the case, at least for the seven lowest levels of the doublet states above $1_{11}$, specifically for collisional transfer of molecules from the lower levels of the doublet states to any of the upper levels of the doublet states (red diamonds). It can therefore be concluded that collisions are the mechanism responsible for population transfer from the lower to the upper levels of the doublet states.

The bottom panel of Fig. 12 shows the corresponding case for the upper levels of the doublet states. Also in this case it is seen that the radiative rates for transitions from the upper levels of the doublet states to the associated lower levels is small compared to radiative rates between the different upper levels
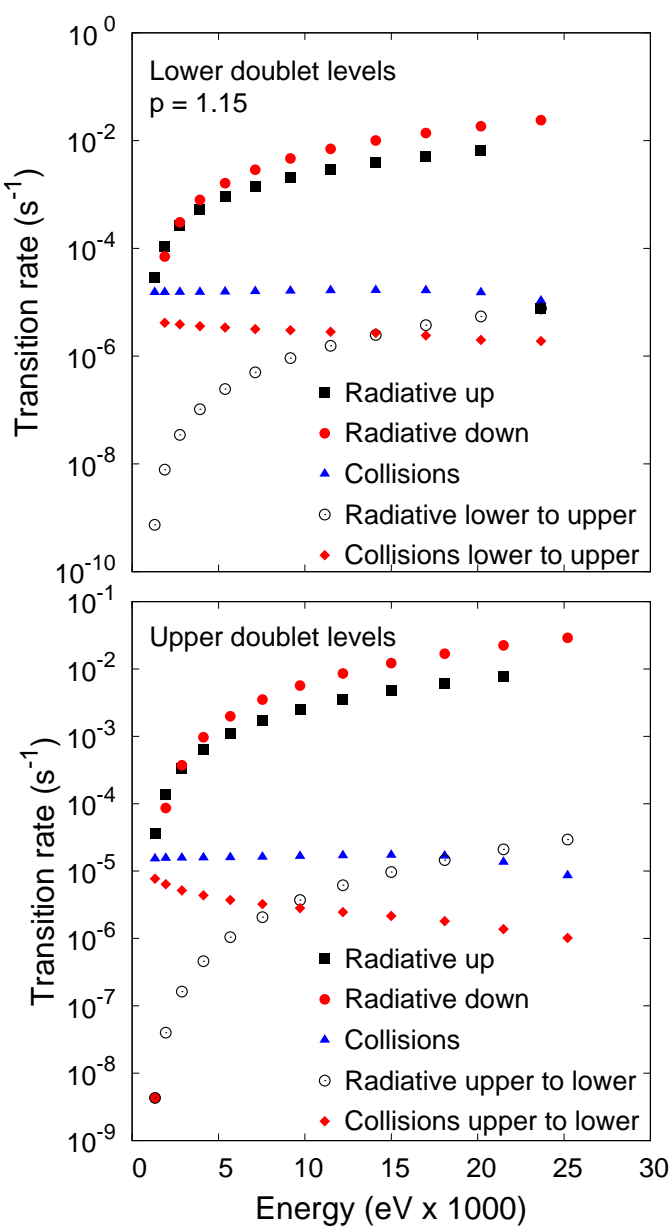

Fig. 12. Radiative and collision rates of the different energy levels for the lower levels of the doublet states (upper panel) and upper levels of the doublet states (bottom panel). The rates were calculated for $T_{\mathrm{d}}=$ $100 \mathrm{~K}, T_{\mathrm{k}}=180 \mathrm{~K}, n_{\mathrm{H}_{2}}=10^{4.25} \mathrm{~cm}^{-3}$, and $p=1.15$. Radiative up (black squares) means upward radiative excitation out of a state (absorption); radiative down (red filled circles) means the sum of spontaneous and stimulated emission rates; collisions (blue triangles) means total collision rate out of a state; radiative lower to upper (open circles) means radiative rate for absorption from the lower level of a doublet state to the associated upper level; collisions lower to upper (red diamonds) means the collision rate from the lower level of a doublet state to its associated upper level.

of the doublet states and the collisional rates from the upper to the lower levels of the doublet states. Both the upper and lower panels show rather clearly that population exchange between the lower and upper levels of the doublet states is preferentially through collisions and not radiation.

Excitation out of $1_{11}$ need not be mainly radiative, but for a given $T_{\mathrm{k}}$ and $n_{\mathrm{H}_{2}}$ it also depends on the energy density in the radiation field at the respective frequencies for the $1_{11} \rightarrow 1_{10}$ $(4.8 \mathrm{GHz})$ and $1_{11} \rightarrow 2_{12}(\sim 140 \mathrm{GHz})$ transitions. In Fig. 13 we show the case when $p=2$, in other words when the SED for $v \ll v_{0}$ (Eq. (2)) is significantly steeper $\left(I_{v} \propto v^{4}\right)$ than for $p=1.15\left(I_{v} \propto v^{3.15}\right)$. It is seen that the upward radiative rates for the lower levels of the doublet states (upper panel, black squares) are significantly lower and for the lower $J$ levels even lower than the collisional rates. Collisional excitation out of $1_{11}$ actually dominates radiative excitation in this case. The radiative rates for the transfer of molecules from the lower levels of the doublet states to any of the upper levels of the doublet states (open 

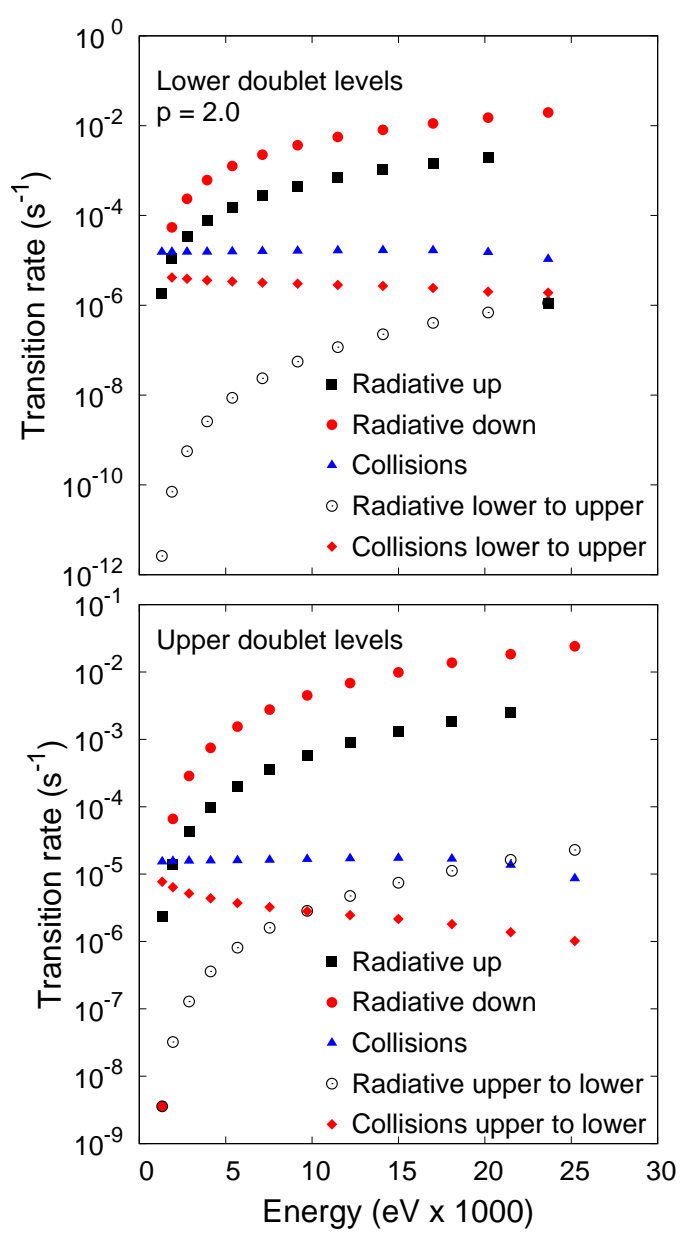

Fig. 13. Same as for Fig. 12, but for $p=2.0$.

circles) are now also significantly smaller compared to the corresponding collision rates (red diamonds). Thus, as for $p=1.15$, population exchange between the lower and upper levels of the doublet states through collisions is by far the dominant process for $p=2$.

A possible pumping scheme is therefore that molecules are radiatively or collisionally excited out of the $1_{11}$ state. Radiative excitation out of $1_{11}$ is almost exclusively to the $2_{12}$ state from where further upward radiative excitations to the lower levels of the doublets can take place. Collisional excitation out of $1_{11}$ can be either to the lower or upper levels of the doublets. Radiative excitation out of $1_{11}$ to $1_{10}$ is very slow, which means that the ladder of lower level doublet states will be populated significantly faster than the ladder of upper level doublet states. A fraction of the population in the lower level doublet states is collisionally transferred to the upper level doublet states, which then provides a relatively fast downward radiative route to $1_{10}$ to create a population inversion. Figure 14 is a schematic representation of the proposed pumping scheme.

The above-proposed pumping scheme can account, at least qualitatively, for the presented results. First, it is clear that without collisions (i.e. with only the internal and external radiation fields present) there can be no effective population transfer from the ladder of lower doublet states to the upper doublet states to eventually overpopulate the $1_{10}$ state relative to the $1_{11}$ state. This explains the absence of an inversion when collisions are switched off. Comparison of the bottom panel of Fig. 9 and both

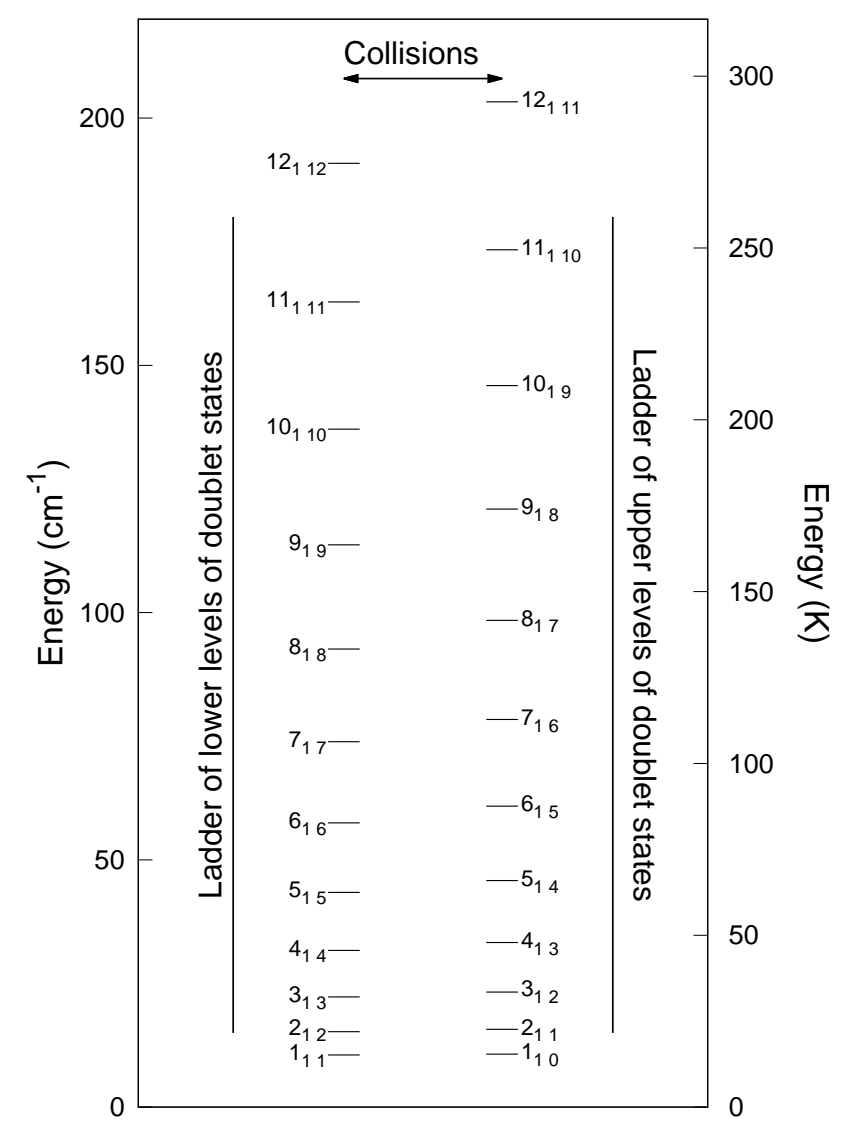

Fig. 14. Energy level diagram of lower levels of the doublets and the upper levels of the doublets to illustrate the proposed pumping scheme for the $4.8 \mathrm{GHz} \mathrm{H} \mathrm{H}_{2} \mathrm{CO}$ masers. Collisions couple the two sets of states.

panels of Fig. 11 shows that the inversion of the $1_{10}-1_{11}$ transition is smaller for $p=0.5$ compared to the cases of $p=1.15$ and $p=2$. Since the energy density in the radiation field varies as $v^{2+p}$ for $v \ll v_{0}$, it follows that for $p=0.5$ the radiative excitation rates out of the lower levels of the doublets is significantly higher than for $p=2$. The upper panel of Fig. 12 also shows that the upward and downward radiative rates for the lower levels of the doublet states are very similar for $p=1.15$. As a consequence, molecules are more or less 'trapped' within the ladder of lower doublet states when $p=0.5$ with the probability to be collisionally transferred to the ladder of upper levels of the doublet states being small. On the other hand, for $p=2$ the downward rates within the ladder of lower level doublet states are markedly higher than the upward rates, meaning that molecules in higher $J$ states will rather quickly radiatively decay to lower states where the probability for collisional transfer to the ladder of upper level doublet states is comparable to that of upward radiative excitation within the ladder of the lower levels of the doublet states. Such a scenario can explain why the inversion is larger for $p=2$ than for $p=0.5$.

\subsection{Constraints due to the $\mathrm{H}_{2} \mathrm{CO}$ abundance}

Figures 8 to 11 suggest that inversion of the $1_{10}-1_{11}$ transition can happen over a significant part of the parameter space with the implication that the $4.8 \mathrm{GHz}$ masers should be quite abundant. However, the $\mathrm{H}_{2} \mathrm{CO}$ masers are very rare, which means that there must be other factors that affect the occurrence of these masers. van der Walt (2014) noted that his results imply 


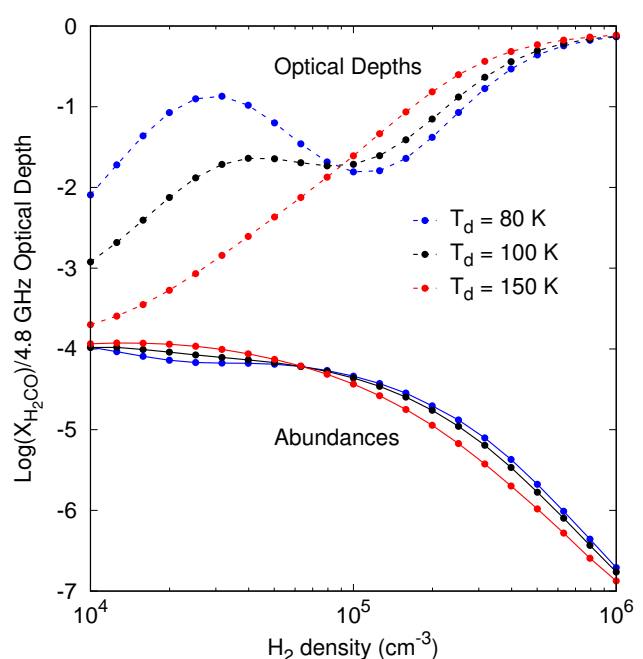

Fig. 15. Plot showing $X_{\mathrm{H}_{2} \mathrm{CO}}$ (solid lines) and $4.8 \mathrm{GHz}$ optical depth (dashed lines) as a function of $\mathrm{H}_{2}$ density for $T_{\mathrm{k}}=180 \mathrm{~K}, p=1.15$, and $W=1$ for different dust temperatures. A maser path length of $10^{17} \mathrm{~cm}$ and line width of $1 \mathrm{~km} \mathrm{~s}^{-1}$ was used to calculate the $\mathrm{H}_{2} \mathrm{CO}$ abundance.

that the $\mathrm{H}_{2} \mathrm{CO}$ abundance must be significantly higher than what is generally observed. In our calculations the $\mathrm{H}_{2} \mathrm{CO}$ abundance, $X_{\mathrm{H}_{2} \mathrm{CO}}$, is implicitly included in the specific column density given by $X_{\mathrm{H}_{2} \mathrm{CO}} n_{\mathrm{H}_{2}} \ell / \Delta v$, where $\ell$ is the maser path length and $\Delta v$ the maser line width. In the variation of $\tau_{4.8}$ with the specific column density (e.g. as in Fig. 2) the most obvious specific column density that can be used to calculate the abundance is that where the optical depth has its maximum negative value. Figure 15 shows, as an example, the variation of the optical depth and $X_{\mathrm{H}_{2} \mathrm{CO}}$ at $T_{\mathrm{k}}=180 \mathrm{~K}$ for $T_{\mathrm{d}}=80,100,150 \mathrm{~K}, W=1$ (Figs. 8$11), \Delta v=10^{5} \mathrm{~cm} \mathrm{~s}^{-1}$, and $\ell=10^{17} \mathrm{~cm}$ (Cragg et al. 2002). The variation of the optical depth with $n_{\mathrm{H}_{2}}$ for the three dust temperatures is seen to be quite different. On the other hand, the derived $\mathrm{H}_{2} \mathrm{CO}$ abundances behave in the same way and vary between $\sim 1.0 \times 10^{-4}$ and $\sim 1.3 \times 10^{-7}$ from $n_{\mathrm{H}_{2}}=10^{4} \mathrm{~cm}^{-3}$ to $10^{6} \mathrm{~cm}^{-3}$. Using the filling factor corrected data presented in Table 8 of Ginsburg et al. (2011), an average $n_{\mathrm{H}_{2}}$ of $4.5 \times 10^{4} \mathrm{~cm}^{-3}$ and average $\mathrm{H}_{2} \mathrm{CO}$ abundance of $1.1 \times 10^{-11}$ for more than 60 lines of sight were found. It is clear that the observed abundances are orders of magnitude too small to explain the masers. The required abundances are significantly higher than most models can produce (see e.g. Guzmán et al. 2011). It is worth noting, however, that Maret et al. (2004) derived a $\mathrm{H}_{2} \mathrm{CO}$ abundance of $6 \times 10^{-6}$ for the warm inner envelope of the young low mass star L1527. This suggests that similar cases may also exist for young high mass stars.

Recently Vichietti et al. (2016) proposed a chemical route for the production of $\mathrm{H}_{2} \mathrm{CO}$ in young high mass star forming regions leading to abundances as high as $10^{-5}$. Using this as a guide, we illustrate in Fig. 16 what the effect is of setting an upper limit of $10^{-5}$ for the $\mathrm{H}_{2} \mathrm{CO}$ abundance on the region in the $n_{\mathrm{H}_{2}}-T_{\mathrm{k}}$ plane where an inversion can occur. Comparison with Fig. 9 shows that the allowed region for an inversion is significantly smaller than when no constraint is put on the $\mathrm{H}_{2} \mathrm{CO}$ abundance. Such a significantly smaller region in the $n_{\mathrm{H}_{2}}-T_{\mathrm{k}}$ plane might be a reason for the rarity of the $\mathrm{H}_{2} \mathrm{CO}$ masers.

An important aspect of Fig. 16 is that for $W=0.3$ (upper panel) inversion of the $1_{10}-1_{11}$ transition occurs basically for $n_{\mathrm{H}_{2}}>10^{5.5} \mathrm{~cm}^{-3}$. Within the framework of our calculations the implication is that the collision rates as shown in Figs. 12 and 13

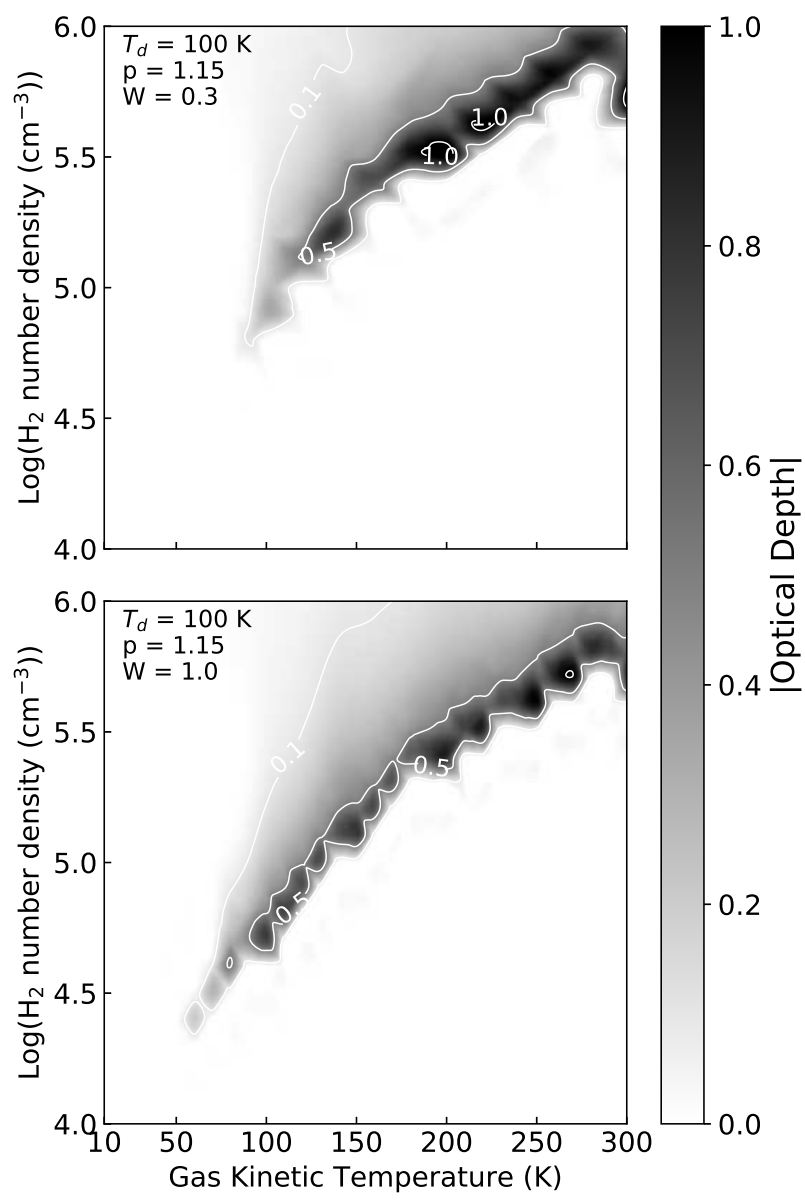

Fig. 16. Variation of $\left|\tau_{4.8}\right|$ in the $n_{\mathrm{H}_{2}}-T_{\mathrm{k}}$ plane, with the SED of the dust emission given by Eq. (2), with $p=1.15$ and $T_{\mathrm{d}}=100 \mathrm{~K}$ and when the $\mathrm{H}_{2} \mathrm{CO}$ abundance is restricted to be less than $10^{-5}$. The upper panel is for $W=0.3$ and the lower panel for $W=1$. This result should be compared with that shown in Fig. 9 where no upper limit is placed on the $\mathrm{H}_{2} \mathrm{CO}$ abundance.

(blue triangles and red diamonds) have to be increased by a factor of $1.25 \mathrm{dex}$. If this is done the collisional excitation rate out of $1_{11}$ is seen to dominate the radiative excitation rate by more than an order of magnitude. If this is the case it can indeed be said that the $\mathrm{H}_{2} \mathrm{CO}$ masers are collisionally pumped. In fact, this explains why there is a region in the $n_{\mathrm{H}_{2}}-T_{\mathrm{k}}$ plane where an inversion can occur without the presence of an external radiation field.

\subsection{Evaluation of the results of Baan et al. (2017)}

As is shown in Sect. 4.1, using the same set of parameter values as was used by Baan et al. (2017), the population inversion became weaker when $T_{\mathrm{k}}$ approached $T_{\mathrm{d}}$ and disappeared completely when $T_{\mathrm{k}}=T_{\mathrm{d}}$. As we show in Fig. 3, the disappearance of the population inversion is most definitely due to the system being in thermodynamic equilibrium when $T_{\mathrm{k}}=T_{\mathrm{d}}$ and that a population inversion is not possible in such a case. It can also be seen, especially from Figs. 9 and 10, that the condition $T_{\mathrm{k}}<T_{\mathrm{d}}$ as a requirement for an inversion, as set by Baan et al. (2017) (and not by van der Walt 2014, as stated by these authors), does not follow from our results.

The results presented above have some definite implications for interpreting the $\mathrm{H}_{2} \mathrm{CO}$ megamaser emission presented by Baan et al. (2017). It is first necessary to note that no inversion is found for $T_{k} \lesssim 100 \mathrm{~K}$ when using a more realistic SED with 
$T_{\mathrm{d}}=59 \mathrm{~K}$ for the dust emission (Fig. 6). A direct implication of this result is that, contrary to the conclusions by Baan et al. (2017), the $4.8 \mathrm{GHz} \mathrm{H}_{2} \mathrm{CO}$ megamasers in the three starburst galaxies are not associated with cold material. Furthermore, if the effect of an upper limit of $10^{-5}$ on the abundance of o$\mathrm{H}_{2} \mathrm{CO}$ is taken into account (Fig. 16), the implication is that the masers trace hot $(100-300 \mathrm{~K})$ gas with densities $10^{5}-10^{6} \mathrm{~cm}^{-3}$ rather than cold gas. Such kinetic temperatures have recently been reported by Gieser et al. (2021) for quite a number of high mass star forming regions. It is fairly well established that the $4.8 \mathrm{GHz}$ masers in the Galaxy are associated exclusively with high mass star forming regions (Araya et al. 2015). There is thus no reason why it should not also be the case for starburst galaxies. In the three starburst galaxies observed by Baan et al. (2017) the $4.8 \mathrm{GHz}$ maser emission is spatially extended and associated with star forming regions, which by itself suggests the association of these masers with high mass star forming regions as in the case of the Galaxy. Why $\mathrm{H}_{2} \mathrm{CO}$ maser emission is associated with only three starburst galaxies, and exactly why it is rare in the Galaxy, are questions still to be answered.

\section{Conclusions}

We performed a new set of numerical calculations to investigate the inversion of the $1_{10}-1_{11}$ transition of $\mathrm{o}-\mathrm{H}_{2} \mathrm{CO}$. The calculations covered a significantly larger part of parameter space compared to previous calculations. Considering the results presented above we summarize and conclude as follows:

1. While theoretically there is a population inversion of the $1_{10}-1_{11}$ transition when using a $50 \mathrm{~K}$ black-body radiation field, the decrease in the optical depth as the kinetic temperature is increased from 10 to $40 \mathrm{~K}$, as presented if Fig. 19 of Baan et al. (2017), is due to the system approaching thermodynamic equilibrium. This behaviour does not imply that the inversion is the consequence of far-infrared pumping. When a more realistic grey-body SED corresponding to that of Arp 220 is used, no inversion of the $1_{10}-1_{11}$ transition is found for $T_{\mathrm{k}}<100 \mathrm{~K}$. Our calculations suggest that the megamasers in the three starburst galaxies observed by Baan et al. (2017) are not associated with cold material, but rather with dense and hot gas associated with high mass star formation.

2. We show that, within the framework of our calculations, the $1_{10}-1_{11}$ transition can be inverted without the presence of a far-infrared radiation field. On the other hand, no evidence could be found for the $1_{10}-1_{11}$ transition being inverted by the external dust radiation field (i.e. when collisions are switched off). Collisions therefore seems to play a key role in the pumping mechanism of the masers.

3. We proposed a pumping scheme in which molecules are excited either radiatively or collisionally out of the $1_{11}$ state. Radiative excitation out of $1_{11}$ leads to the population of the ladder of lower levels of the doublet states. Population exchange between the ladders of lower and upper doublet states is collisional since radiative coupling between the two ladders is weak. At high $\mathrm{H}_{2}$ densities collisional excitation out of $1_{11}$ can be to both ladders. Molecules excited collisionally to higher states in the ladder of lower levels of the doublet states still have to be transferred collisionally to the ladder of upper levels of the doublet states. Without collisions, population exchange between the ladders of the lower and upper levels of the doublet states is extremely slow. Transfer of molecules from the lower levels of the doublet states to the upper levels of the doublet states is necessary since it then provides a fast radiative decay route to $1_{10}$.

4. When assuming reasonable values for the maser line width and the maser path length it is found that, over most of parameter space where theoretically an inversion can occur, the required abundance of $\mathrm{H}_{2} \mathrm{CO}$ is significantly larger than observed and predicted by models. Limiting the $\mathrm{H}_{2} \mathrm{CO}$ abundance to less than $10^{-5}$ significantly reduces the region in the $n_{\mathrm{H}_{2}}-T_{\mathrm{k}}$ plane where an inversion can occur to a rather narrow region with $\mathrm{H}_{2}$ densities greater than $\sim 10^{5} \mathrm{~cm}^{-3}$ and $T_{\mathrm{k}} \gtrsim$ $100 \mathrm{~K}$. This might explain the rarity of the $\mathrm{H}_{2} \mathrm{CO}$ masers in the Galaxy and starburst galaxies.

We finally point out that $\mathrm{o}-\mathrm{H}_{2} \mathrm{CO}$ has several submillimeter transitions between excited states (for which $E_{\text {upper }}>100 \mathrm{~K}$ ) which can be used to probe the physical conditions of the environments of the $\mathrm{H}_{2} \mathrm{CO}$ masers. Dedicated high resolution observations of these transitions can be used to test the predictions of our model calculations.

Acknowledgements. This work is based on research supported in part by the National Research Foundation of South Africa (Grant Numbers 132494).

\section{References}

Araya, E., Baan, W. A., \& Hofner, P. 2004, ApJS, 154, 541

Araya, E. D., Hofner, P., Goss, W. M., et al. 2008, ApJS, 178, 330

Araya, E. D., Olmi, L., Morales Ortiz, J., et al. 2015, ApJS, 221, 10

Baan, W. A., Guesten, R., \& Haschick, A. D. 1986, ApJ, 305, 830

Baan, W. A., An, T., Klöckner, H.-R., \& Thomasson, P. 2017, MNRAS, 469, 916

Barbosa, C. L., Blum, R. D., Damineli, A., Conti, P. S., \& Gusmão, D. M. 2016, ApJ, 825, 54

Boland, W., \& de Jong, T. 1981, A\&A, 98, 149

Cragg, D. M., Sobolev, A. M., \& Godfrey, P. D. 2002, MNRAS, 331, 521

Downes, D., \& Eckart, A. 2007, A\&A, 468, L57

Gieser, C., Beuther, H., Semenov, D., et al. 2021, A\&A, 648, A66

Ginsburg, A., Darling, J., Battersby, C., Zeiger, B., \& Bally, J. 2011, ApJ, 736, 149

Ginsburg, A., Walsh, A., Henkel, C., et al. 2015, A\&A, 584, L7

Green, J. A., Breen, S. L., Fuller, G. A., et al. 2017, MNRAS, 469, 1383

Guzmán, V., Pety, J., Goicoechea, J. R., Gerin, M., \& Roueff, E. 2011, A\&A, 534, A49

Kraemer, K. E., Jackson, J. M., Deutsch, L. K., et al. 2001, ApJ, 561, 282

Lim, W., \& De Buizer, J. M. 2019, ApJ, 873, 51

Lu, X., Mills, E. A. C., Ginsburg, A., et al. 2019, ApJS, 244, 35

Maret, S., Ceccarelli, C., Caux, E., et al. 2004, A\&A, 416, 577

Nikitin, A. V., Protasevich, A. E., Rodina, A. A., et al. 2021, J. Quant. Spectr. Rad. Transf., 260, 107478

Schöier, F. L., van der Tak, F. F. S., van Dishoeck, E. F., \& Black, J. H. 2005, A\&A, 432, 369

Scoville, N., Sheth, K., Walter, F., et al. 2015, ApJ, 800, 70

Scoville, N., Murchikova, L., Walter, F., et al. 2017, ApJ, 836, 66

van der Tak, F. F. S., Black, J. H., Schöier, F. L., Jansen, D. J., \& van Dishoeck, E. F. 2007, A\&A, 468, 627

van der Walt, D. J. 2014, A\&A, 562, A68

van Langevelde, H. J., \& van der Tak, F. 2008, Radiation Bookkeeping: a guide to astronomical molecular spectroscopy and radiative transfer problems with an emphasis on RADEX, http://var.sron.nl/radex/radex_manual. pdf, [Online; accessed 10 March 2021]

Vichietti, R. M., Spada, R. F. K., da Silva, A. B. F., Machado, F. B. C., \& Haiduke, R. L. A. 2016, ApJS, 225, 2

Wiesenfeld, L., \& Faure, A. 2013, MNRAS, 432, 2573

Wilson, C. D., Rangwala, N., Glenn, J., et al. 2014, ApJ, 789, L36

Yun, M. S., \& Carilli, C. L. 2002, ApJ, 568, 88 